\title{
A Simple Method for the Selection of a Suitable Emulsifier Based on Color Difference
}

\author{
Kenjiro Koga, ${ }^{*, a}$ Yoshiro Ishitobi, ${ }^{a}$ Masaki Imata, ${ }^{b}$ Masahiro Murakami, ${ }^{b}$ and Susumu Kawashima ${ }^{a}$ \\ ${ }^{a}$ Faculty of Pharmaceutical Sciences, Hokuriku University; Ho-3, Kanagawa-machi, Kanazawa 920-1181, Japan: and \\ ${ }^{b}$ Discovery Research Laboratory, Laboratories of Excellent Medicine, Amato Pharmaceutical Products; 995 Sasao-cho, \\ Fukuchiyama 620-0932, Japan. Received August 1, 2002; accepted September 9, 2002
}

\begin{abstract}
The purpose of this study was to establish a simple and rapid method for selecting a nonionic surfactant to prepare an emulsion that can maintain a stable emulsification phase. As an index of the degree of emulsification, the white chromaticity of the prepared sample was measured using a color difference meter. When liquid paraffin was used as an oil, the color difference $(\mathrm{dE}(\mathrm{H}))$ was shown as a change in a $\mathrm{V}$-shaped curve depending on changes in the hydrophile-lipophile balance (HLB) number of sorbitan ester, polyoxyethylene sorbitan ester, or polyethyleneglycol ester. Although there was a difference in the minimal value of $\mathrm{dE}(\mathrm{H})$ in these experiments, the dE(H) was similar to the required HLB of liquid paraffin $(H L B=10)$. On the other hand, no relationship was found between the HLB number and color difference when polyglycerol ester was used. It seems that there was possibility of the effect by the lowering of apparent HLB with a collapse of the hydration. These results indicate that color difference could be utilized for the selection of surfactants, except for polyglycerol ester.
\end{abstract}

Key words nonionic surfactant; emulsion; color difference; hydrophile-lipophile balance

Emulsion pharmaceutical preparation is relevant to a wide range of factors, including improvement of the solubility of therapeutic agents, improvement of stability, drug delivery system carriers, etc. For safety, nonionic surfactants are mainly used as emulsifying agents. Based on the material characteristics, the type of surfactant is often chosen depending on experience. In the process of emulsification, a balance of hydrophilicity and hydrophobicity in the emulsifying agent is important. Therefore, the concept of hydrophilelipophile balance (HLB) was advocated. ${ }^{1,2)} \mathrm{A}$ few years later, an organic conceptual scheme method ${ }^{3)}$ and an emulsion inversion point method ${ }^{4)}$ were proposed for the selection of an emulsifying agent. In these methods, the HLB method of Griffin is the most convenient in the selection of an emulsifying agent. Furthermore, it was shown that a surfactant had to have the optimum HLB number to obtain the best emulsification conditions for the type of emulsifying oil used. ${ }^{5)}$ Lin et $a l$. reported the relationship between the HLB number of the surfactant and the solubilization quantity of water or emulsification particle size as follows. ${ }^{6}$ ) When polyoxyethylene oleyl ether $(\mathrm{HLB}=10)$ or mixture $(\mathrm{HLB}=10)$ of Span 80 and Tween 80 was used as an emulsifying agent, the emulsification particle size was small (approximately $2 \mu \mathrm{m}$ ) and the solubilization quantity of water in the emulsion was at the maximum. In those original reports, it was assumed that small emulsification particle size contribute to superior emulsification conditions, and that more particles change to superior white emulsion. In the present study, we discuss the utility of color measuring from the relationship between the HLB number of several types of surfactant and the $\mathrm{dE}(\mathrm{H})$ value of the emulsion.

\section{MATERIALS AND METHODS}

Materials Liquid paraffin was obtained from Wako Pure Chemical Industries (Osaka, Japan). Methylene blue was from Nippon Soda (Tokyo, Japan). The nonionic surfactants used as emulsifying agents are shown in Table 1. Span 20, Tween 60, Tween 80, and Tween 85 were purchased from
Wako Pure Chemical Industries. Tween 21 and Tween 81 were from Sigma Chemical (St. Louis, MO, U.S.A.). Span 30 and Span 80 were gifts from Daiichi Kogyo Seiyaku (Kyoto, Japan). Polyethyleneglycol esters and polyglycerol esters were gifts from Nikko Chemicals (Osaka, Japan) and Sakamoto Yakuhin Kogyo (Osaka, Japan), respectively.

Preparation of Emulsion One-half gram of one or two surfactant(s) was placed in a $100-\mathrm{ml}$ vial, $5.00 \mathrm{~g}$ of liquid paraffin and a magnetic stir bar were added to the vial, and it was mixed at $70^{\circ} \mathrm{C}$ at $200 \mathrm{rpm}$ for $10 \mathrm{~min}$ using a hot plate stirrer DP-1L (Iuchi, Tokyo, Japan). Methylene blue solution $1.0 \mathrm{~g}, 0.001 \%(\mathrm{w} / \mathrm{v})$, was added to the emulsion, and it was mixed under the same conditions for $20 \mathrm{~min}$. After the vial was removed from the stirrer, $50 \mathrm{~g}$ of $0.001 \%(\mathrm{w} / \mathrm{v})$ methylene blue solution was added to the vial and the vial was

Table 1. Nonionic Surfactants and Their Physicochemical Properties

\begin{tabular}{llc}
\hline \hline \multicolumn{1}{c}{ Chemical name } & \multicolumn{1}{c}{$\begin{array}{c}\text { Product } \\
\text { name }\end{array}$} & $\begin{array}{c}\text { HLB Type } \\
\text { number }\end{array}$ \\
\hline Sorbitan monolaurate & Span 20 & $8.6 \mathrm{o} / \mathrm{w}$ \\
Sorbitan sesquioleate & Span 30 & $3.7 \mathrm{w} / \mathrm{o}$ \\
Sorbitan monooleate & Span 80 & $4.3 \mathrm{w} / \mathrm{o}$ \\
Polyoxyethylene(6)sorbitan monolaurate & Tween 21 & $13.3 \mathrm{o} / \mathrm{w}$ \\
Polyoxyethylene(6)sorbitan monooleate & Tween 81 & $10.0 \mathrm{o} / \mathrm{w}$ \\
Polyoxyethylene(20)sorbitan monostearate & Tween 60 & $14.9 \mathrm{o} / \mathrm{w}$ \\
Polyoxyethylene(20)sorbitan monooleate & Tween 80 & $15.0 \mathrm{o} / \mathrm{w}$ \\
Polyoxyethylene(20)sorbitan trioleate & Tween 85 & $11.0 \mathrm{o} / \mathrm{w}$ \\
Polyethyleneglycol(10EO)monostearate & MYS-10 & $11.0 \mathrm{o} / \mathrm{w}$ \\
Polyethyleneglycol(10EO)monolaurate & MYL-10 & $12.5 \mathrm{o} / \mathrm{w}$ \\
Polyethyleneglycol(25EO)monostearate & MYS-25 & $15.0 \mathrm{o} / \mathrm{w}$ \\
Polyethyleneglycol distearate & CDS-400 & $8.5 \mathrm{o} / \mathrm{w}$ \\
Polyethyleneglycol diisostearate & CDIS-400 & $9.5 \mathrm{o} / \mathrm{w}$ \\
Tetraglycerol monooleate & MO-310 & $10.2 \mathrm{o} / \mathrm{w}$ \\
Hexaglycerol monooleate & MO-500 & $12.2 \mathrm{o} / \mathrm{w}$ \\
Tetraglycerol monolaurate & ML-310 & $11.9 \mathrm{o} / \mathrm{w}$ \\
Tetraglycerol monostearate & MS-310 & $10.2 \mathrm{o} / \mathrm{w}$ \\
Hexaglycerol sesquistearate & SS-500 & $10.1 \mathrm{o} / \mathrm{w}$ \\
Decaglycerol tristearate & TS-750 & $9.1 \mathrm{o} / \mathrm{w}$ \\
& &
\end{tabular}

a) The abbreviations o/w and w/o indicate oil-in-water and water-in-oil emulsification, respectively. The emulsification types prepared with two surfactants in the experiments are $\mathrm{o} / \mathrm{w}$. 
shaken strongly by hand for $30 \mathrm{~s}$. The vial allowed to stand for $1 \mathrm{~h}$ at room temperature.

Color Difference Measurement After a vial with emulsion was shaken by turning over two times, $3 \mathrm{ml}$ of the emulsification phase was placed in the cell of a color measuring system ( 290 , Nippon Denshoku, Tokyo, Japan; SZ optical sensor, Nippon Denshoku) and the brightness and chromaticity of the emulsion were measured. The color specification system has been used as the method for quantitating color measurement. The color difference $(\mathrm{dE}(\mathrm{H}))$ is analyzed in terms of lightness, hue, and chroma components. ${ }^{7,8)}$ In general, the National Bureau of Standards scale has been used as the $\mathrm{dE}(\mathrm{H})$.

Curve Fitting For the relationship between the HLB number of surfactants and $\mathrm{dE}(\mathrm{H})$ value in the emulsion, the curve was fitted using the software program, Excel 2002 (Microsoft).

Viscosity Measurement The viscosity of a prepared emulsion was measured using a viscometer (B-type viscometer BM, Tokyo Keiki, Tokyo, Japan).

\section{RESULTS AND DISCUSSION}

To determine the emulsification conditions of liquid paraffin, the emulsion was prepared with Span 20, Tween 81, Tween 85, Tween 21, and Tween 60 , and then the $\mathrm{dE}(\mathrm{H})$ was measured (Fig. 1).

Methylene blue is a water-soluble pigment and is included in the water phase in emulsions. Therefore a stable emulsification exhibits a while color. On the other hand, in unstable conditions, it appears blue. The $\mathrm{dE}(\mathrm{H})$ value reflects the difference between colors as a distance with the position of the white color as a standard. ${ }^{7)}$ The $\mathrm{dE}(\mathrm{H})$ value slowly decreased with the increase in the HLB number $(<12)$, and abruptly increased with the increase in the HLB number $(>12)$. When Tween 85 or Tween 21 was used, both $\mathrm{dE}(\mathrm{H})$ values were minimum. Therefore the HLB number that has the minimal $\mathrm{dE}(\mathrm{H})$ value was 12.2 from the curve of the graph. It is reported that the required HLB for liquid paraffin is approximately 10.6) As shown in Fig. 1, the HLB value obtained was 12.2, which is higher by more than 2 compared with the reported value $(\mathrm{HLB}=10)$. It appears that the HLB number with the minimal $\mathrm{dE}(\mathrm{H})$ value resulted in a color difference measurement slightly different from that obtained by the method of Lin et al. $^{6 \text { ) }}$

To understand in detail the relationship between the HLB number of surfactant and the $\mathrm{dE}(\mathrm{H})$ value in emulsion, the $\mathrm{dE}(\mathrm{H})$ value was measured at different ratios of the two surfactants sorbitan ester and polyoxyethylene sorbitan ester (Fig. 2). The HLB number of mixture components was determined by calculation of the weight ratio of both surfactants. First, for the use of Span $30(\mathrm{HLB}=3.7)$ and Tween 80 $(\mathrm{HLB}=15.0)$, the HLB number with minimal $\mathrm{dE}(\mathrm{H})$ was 9.3 and white turbidity in the emulsion was very strong. The emulsion conditions of mixture components adjusted to HLB $\gtrsim 10.5$ were all blue in color, suggesting that the production of the emulsion with quite uniform droplet size distribution was slight. Next, for the use of Span $80($ HLB = 4.3) and Tween $85(\mathrm{HLB}=11.0)$, the HLB number with minimal $\mathrm{dE}(\mathrm{H})$ was 9.9. This HLB number was identical to the required HLB of liquid paraffin in the report of Lin et al. ${ }^{6}$
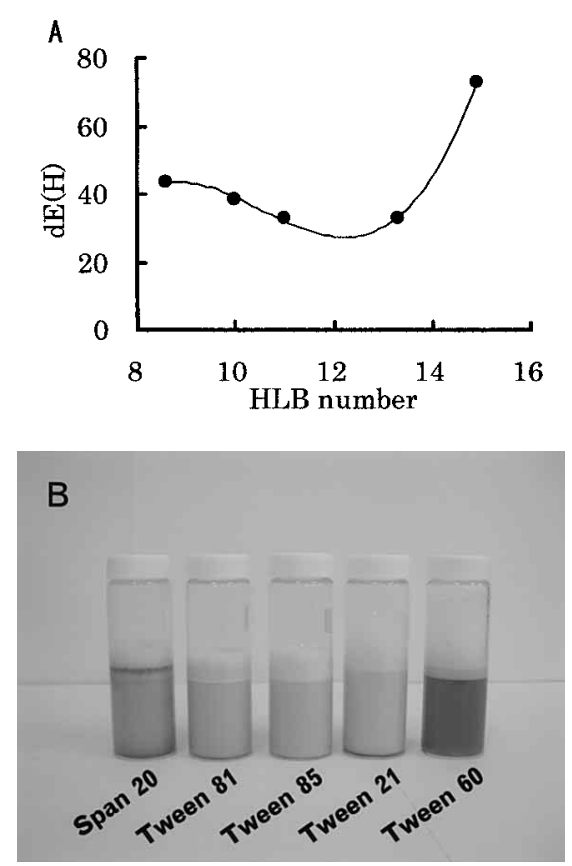

Fig. 1. Relationship between the HLB Number of Surfactants and Color Difference $(\mathrm{dE}(\mathrm{H}))$ in Emulsions of Surfactant/Liquid Paraffin/Water $(0.5: 5: 51, \mathrm{w} / \mathrm{w})(\mathrm{A})$ and Their Appearance (B)

Surfactants used were Span $20(\mathrm{HLB}=8.6)$, Tween $81(\mathrm{HLB}=10.0)$, Tween 85 $(\mathrm{HLB}=11.0)$, Tween $21(\mathrm{HLB}=13.3)$, and Tween $60(\mathrm{HLB}=14.9)$. Each point represents the mean of three experiments. The standard error bar of each point was smaller than the width of symbols. The curve of the graph was drawn according to the third function.

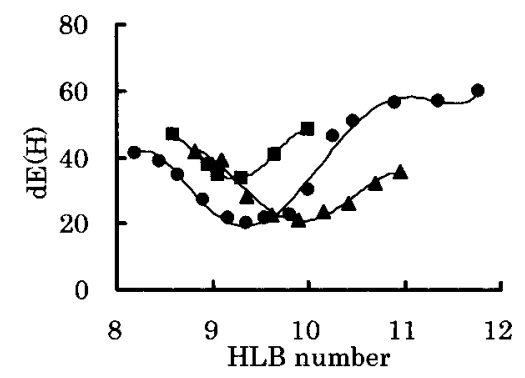

Fig. 2. Relationship between the HLB Number of Surfactants and Color Difference $(\mathrm{dE}(\mathrm{H}))$ in Emulsions of Surfactant/Liquid Paraffin/Water $(0.5: 5: 51, \mathrm{w} / \mathrm{w})$

-: The surfactant was prepared using various mixture ratios of Span 30 and Tween 80; $\boldsymbol{\Lambda}$ : the surfactant was prepared using various mixture ratios of Span 80 and Tween $85 ; \mathbf{\square}$ : the surfactant was prepared using various mixture ratios of Span 20 and Tween 81. The HLB number of each surfactant is shown in Table 1. The HLB numbers of all samples were calculated using the weight ratios of surfactants used. Each point represents the mean of three experiments. The standard error bar of each point was smaller than the width of symbols. The curves for the three symbols were drawn according to the fifth function for closed circles and the fourth function for closed triangles and closed squares.

Moreover, the relationship between the HLB number and the $\mathrm{dE}(\mathrm{H})$ value showed the change with HLB number 9.9 was almost at the left-right symmetry center. It is suggested that the result of color difference with the addition of two surfactants had a similar change pattern to the result reported by Lin et $a l .{ }^{6)}$ Since the HLB numbers with minimal $\mathrm{dE}(\mathrm{H})$, i.e., 9.3 for Span 30/Tween 80 and 9.9 for Span 80/Tween 85, were similar, the relationship of both parameters with the addition of one or two component(s) of Span $20(\mathrm{HLB}=8.6)$ and Tween $81(\mathrm{HLB}=10.0)$ was examined. The HLB with minimal $\mathrm{dE}(\mathrm{H})$ value was 9.3. From these results, the 


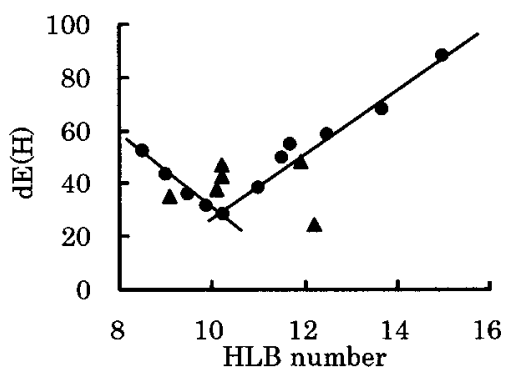

Fig. 3. Relationship between HLB Number of Surfactants and Color Difference $(\mathrm{dE}(\mathrm{H}))$ in Emulsions of Surfactant/Liquid Paraffin/Water $(0.5: 5: 51, \mathrm{w} / \mathrm{w})$

-: The surfactants prepared were CDS-400 (HLB $=8.5)$, CDS-400/CDIS-400 $(1: 1$, $\mathrm{w} / \mathrm{w})(\mathrm{HLB}=9.0)$, CDIS-400 (HLB $=9.5)$, CDIS-400/MYS-10 $(3: 1, \mathrm{w} / \mathrm{w})(\mathrm{HLB}=9.9)$, CDIS-400/MYS-10 $(1: 1, \mathrm{w} / \mathrm{w})(\mathrm{HLB}=10.3)$, MYS-10 (HLB $=11.0)$, MYS-10/MYL$10(2: 1, \mathrm{w} / \mathrm{w})(\mathrm{HLB}=11.5)$, MYS-10/MYL-10 $(1: 1, \mathrm{w} / \mathrm{w})(\mathrm{HLB}=11.7)$, MYL-10 $(\mathrm{HLB}=12.5)$, MYL-10/MYS-25 (1:1, w/w) $(\mathrm{HLB}=13.7)$, and MYS-25 (HLB=15.0). $\boldsymbol{\Lambda}$ : The surfactants were prepared using TS-750, SS-500, MO-310, MS-310, ML-310, or MO-500. Their HLB numbers are shown in Table 1. Each point represents the mean of three experiments for polyethyleneglycol esters and six experiments for polyglycerol esters. The standard error bar of each point was smaller than the width of symbols. The curve for the closed circle was drawn using the least squares method bordering on HLB 10

changes in color difference with the addition of sorbitan ester and polyxyethylene sorbitan ester had a gentle V-shaped curve and the HLB with minimal $\mathrm{dE}(\mathrm{H})$ was the range of 910 . The change in white turbidity in the emulsion was clearly discernible catch visually.

It is difficult to explain why the HLB number with minimal $\mathrm{dE}(\mathrm{H})$ with the addition of two surfactants (Fig. 2) was different from that with the addition of one surfactant (Fig. 1). As a reasonable speculation, however, it is believed that there is a difference in the dehydration of water molecules in the circumference of the oxyethylene chain, and therefore the HLB number appeared to deviate to the hydrophobic side. Although a fine difference was observed for the HLB number with minimal $\mathrm{dE}(\mathrm{H})$ value with the addition of surfactants, it is considered that a color difference measurement becomes the key to distinguishing the stability of an emulsion from the visual changes in an emulsification phase based on the dispersion state of oil. In particular, the difference in the $\mathrm{dE}(\mathrm{H})$ value was clearly reflected with changes at more than 0.3 when the HLB number range was $8-11$. Therefore it appears possible to evaluate the condition of the emulsion preparation by simply using a color difference meter.

The next aim was to clarify whether the relation between the HLB number and $\mathrm{dE}(\mathrm{H})$ value in the combination of sorbitan ester and polyoxyethylene sorbitan ester is similar when a surfactant with a different hydrophilic group was used. Polyethyleneglycol ester and polyglycerol ester were used as emulsifying agents, and then the $\mathrm{dE}(\mathrm{H})$ value in the emulsion was measured (Fig. 3). When polyethyleneglycol ester was used, the HLB number with minimal $\mathrm{dE}(\mathrm{H})$ was 10.2 . As an interesting result, the relationship between the HLB number and $\mathrm{dE}(\mathrm{H})$ was rectilinear in both directions from the boundary point of $\mathrm{HLB}=10.2$. This curve was different from that obtained with the addition of sorbitan and polyoxyethylene sorbitan esters, suggesting that the $\mathrm{dE}(\mathrm{H})$ value with the addition of polyethyleneglycol ester is closely related to the change in the HLB number. On the other hand, when polyglycerol ester was used, the relationship between the HLB number and the $\mathrm{dE}(\mathrm{H})$ value in emulsion showed a markedly different pattern compared with that seen with the addition of sorbitan, polyoxyethylene sorbitan, or polyethyleneglycol esters. That is, a V-shaped relation could not be recognized. The surfactant that had minimal $\mathrm{dE}(\mathrm{H})$ was MO-500 (HLB= $12.2)$ and the $\mathrm{dE}(\mathrm{H})$ value was 24.7 . However, the $\mathrm{dE}(\mathrm{H})$ of ML-310 (HLB =11.9), close to the HLB number of MO-500, was 48.6. The $\mathrm{dE}(\mathrm{H})$ values of other polyglycerol esters were also high compared to that with the addition of MO-500. It appeared to inhibit the emulsion formation in one or more factor(s). As a result of these factors, an increase in viscosity was assumed because the viscosity of these emulsions with the addition of polyglycerol ester, except for MO-500, was higher (35-44 cPs) than when other surfactants were used $(<15 \mathrm{cPs})$. As some mechanisms caused the curious relationship between the HLB number and $\mathrm{dE}(\mathrm{H})$ in the emulsion with the addition of polyglycerol ester, it appears that the increase in the viscosity of the emulsion inhibited the formation of uniform, small-sized droplets regardless of the HLB number, or that the apparent HLB number decreased with the change in the hydrophobic property of the surfactant. The report by Horiuchi et al. ${ }^{9)}$ supports the latter hypothesis. That is, the viscosity B coefficient of polyoxyethylene cetyl ether $\left(\mathrm{C}_{16}(\mathrm{EO})_{\mathrm{n}}\right)$ with different HLB numbers was markedly decreased at temperatures of more than $50^{\circ} \mathrm{C}$, based on the relationship between the viscosity $\mathrm{B}$ coefficient and temperature. This result indicates that the hydrated layer of polyoxyethylene cetyl ether decreases and then the hydrophobic property increases, i.e., a decrease in HLB number occurs. It appears that the increase in viscosity is dependent on this hydrophobic property.

In conclusion, the $\mathrm{dE}(\mathrm{H})$ value using a color difference meter reflects the difference in the HLB number in the emulsion upon the addition of sorbitan ester, polyoxyethylene sorbitan ester, and polyethyleneglycol ester except for polyglycerol ester with high viscosity. Because the HLB number with minimal $\mathrm{dE}(\mathrm{H})$ deviates slightly with surfactant type, it is necessary to evaluate the emulsification conditions in proportion to the surfactant type. By using a color difference meter, it appears possible to determine the mixture ratio of two surfactants and evaluate the stability of the emulsion generated.

Acknowledgments The authors are grateful to Daiichi Kogyo Seiyaku Co. for valuable technical assistance.

\section{REFERENCES}

1) Griffin W. C., J. Soc. Cosmet. Chem., 1, 311-316 (1949).

2) Griffin W. C., J. Soc. Cosmet. Chem., 5, 249-256 (1954).

3) Fujita A., Kagaku no Ryoiki, 11, 719-725 (1957).

4) Marszall L., Cosmet Perfum., 90, 37-39 (1975).

5) ICI Americas, Inc. "The HLB System," ICI, Wilmington, Delaware, 1984.

6) Lin T. J., Kurihara H., Ohta H., J. Soc. Cosmet. Chem., 26, 121-139 (1975).

7) Sato H., Hirota S., Kondo K., "Analysis Chemistry: Base and Experiment of Instrumental Analysis," Corona, Tokyo, 1974, pp. 283-307.

8) Japan Standards Association (ed.), "JIS Handbook: Color," Japan Standard Association, Tokyo, 1990.

9) Horiuchi T., Jinno T., Kashiwa I., J. Soc. Cosmet. Chem. Jpn., 13, 2528 (1979). 